\title{
Actualización del inventario de quirópteros y refugios en Ceuta: primera cita de Pipistrellus pygmaeus en el norte de África
}

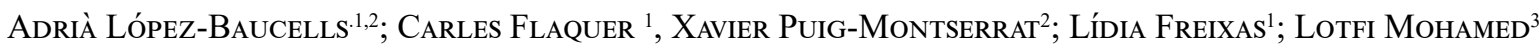 \\ 1. Granollers Museum of Natural Sciences. Bat Research Group (Granollers, Catalunya) \\ 2. Galanthus Association (Celrà, Catalunya) \\ ${ }^{3}$. Instituto de Estudios Ceutíes (Ceuta)
}

Correo electrónico del autor: adria.baucells@gmail.com

DOI: http://dx.doi.org/10.14709/BarbJ.5.1.2012.07

English title: Updated inventory of bats and their refuges in Ceuta: first record of Pipistrellus pygameus in north Africa

\begin{abstract}
Recent knowledge of the bat populations of Ceuta was deficient for both forest and cave-dwelling species, whose refuges had not been correctly monitored in recent years (some of them not visited for 18 years). On these grounds, new surveys have been conducted using mist nets and bat detectors and visits to shelters. Although the number of bats species in Ceuta has been increased by two and two new phonic groups, generally speaking we observed a decline in cave-dwelling bats, with just three breeding colonies of Rhinolophus hipposideros and few or none of the following species: Rhinolophus mehelyi, Rhinolophus euryale, Rhinolophus blasii, Myotis capaccinii and Miniopterus schreibersii. The entrance to La Mina de la Fuente, a roost that had once housed four species of bats, had collapsed and was reopened. Mist netting and bat detector sessions revealed low activity rates. Particularly it is important to highlight the first record of Pipistrellus pygmaeus in Africa. In light of these new surveys we recommend that the roosts of cave-dwelling bats be monitored annually (especially La Mina de la Fuente).
\end{abstract}

Keywords: Ceuta, roosts, Cave dwelling bats, Pipistrellus pygmaeus, Myotis punicus, Rhinolophus hipposideros , ultrasounds, bat conservation

Resumen: El conocimiento de las poblaciones de quirópteros de la Ciudad Autónoma de Ceuta era claramente deficiente en ciertos aspectos, sobre todo en relación a las especies de tipo forestal, pero también en las revisiones realizadas de los refugios de la zona, que presentan vacíos temporales de hasta 18 años. Por estos motivos se han realizado nuevos muestreos en bosque, en embalses y charcas mediante redes de niebla, así como noches de detección automática mediante detectores de alta velocidad, y se ha realizado una revisión exhaustiva de los refugios conocidos y potenciales que pudieran albergar colonias de quirópteros. Aunque se ha ampliado el inventario en 2 especies y 2 grupos fónicos, se ha determinado un descenso relativo en la abundancia de ejemplares en los refugios (con solamente citas reproductivas de Rhinolophus hipposideros en 3 refugios), así como una disminución acusada de las especies cavernícolas presentes en el área (no se ha detectado Rhinolophus mehelyi, Rhinolophus euryale, Rhinolophus blasii, Myotis capaccinii ni Miniopterus schreibersii). La Mina de la Fuente, que albergaba 4 especies cavernícolas, fue localizada con la entrada derrumbada y fue reabierta nuevamente. Mediante las sesiones de capturas y la detección con detectores se han obtenido unos índices de actividad cmuy bajos Entre las nuevas citas para el área (Tadarida teniotis etc.) destaca la de Pipistrellus pygmaeus primera referencia de la especie en el continente africano. Tras estas nuevas prospecciones, se considera importante realizar una revisión anual completa de refugios protocolizada y constante para determinar la tendencia de las colonias de cría de R. hipposideros y la posible recolonización de otras especies (en especial en la Mina de la Fuente).

Palabras clave: Ceuta, refugios, quirópteros cavernícolas, Pipistrellus pygmaeus Myotis punicus, Rhinolophus hipposideros, detección ultrasonidos, conservación 


\section{INTRODUCCIÓN}

La Ciudad Autónoma de Ceuta, dada su situación geográfica, en la vertiente Africana del estrecho de Gibraltar, su zona costera y su clima mediterráneo presenta un conjunto de características que hace de sus espacios naturales unos ecosistemas realmente interesantes en cuanto a su funcionalidad ecológica para diversos grupos de organismos y el papel de barrera geográfica del mismo Estrecho. Los espacios naturales que se encuentran en esta área forman parte de la Red Natura 2000 y corresponden al Lugar de Importancia Comunitaria (LIC) - Zona de Especial Protección para las Aves (ZEPA) CalamocarroBenzú (ES6310001), ZEPA Acantilados del Monte Hacho (ES0000197) y el LIC Zona Marítimo Terrestre del Monte Hacho (ES6310002). La zona de la Ciudad Autónoma de Ceuta ha obtenido un reconocimiento importante por su papel como cuello de botella en las migraciones latitudinales de aves. Hasta 136 especies cruzan el Estrecho de Gibraltar por esta zona, de las cuales 32 se encuentran en el Anexo I de la Directiva 79/409/CEE. Miles de ejemplares, más de 5000 rapaces, etc. cruzan y utilizan esta área como zona de reposo y alimentación durante sus migraciones pre y postnupciales (Viada 1998). Paralelamente, esta zona se ha caracterizado por la instalación de varias estructuras y edificios militares que, tras su abandono, han ofrecido refugio a ciertas especies de murciélagos de tipo cavernícola, algunas de las cuales se encuentran amenazadas según la clasificación de la UICN 2006.

Se han realizado varios estudios quiropterológicos por parte de diversos equipos científicos desde inicios de los años 90, centrados básicamente en la revisión de refugios (SerraCobo 1993, Castilla 2000, Ibáñez C. 2004). De todos modos, no existe ningún seguimiento a largo plazo de los refugios y las colonias que crían o se refugian en los territorios de Ceuta, y los datos existentes al respecto son dispersos y fragmentados, con intervalos de réplicas de hasta 18 años en algunos casos. En algunos casos, los mismos refugios han sido derruidos o cerrados sin ningún tipo de seguimiento. Por otra parte, la mayoría de los estudios realizados en Ceuta hasta la fecha se centraron única y exclusivamente en los quirópteros cavernícolas del género Rhinolophus, o especies como Miniopterus schreibersii o algunos Myotis sp. Consecuentemente, el inventario de quirópteros de la zona era claramente deficiente, con solamente una cita de Pipistrellus pipistrellus y un vacío importante en relación a murciélagos fisurícolas y forestales. En general a modo de revisión bibliográfica, existen pocos inventarios forestales en la zona norte de África, y la mayoría se centran igualmente en especies cavernícolas (Lieron 2008, Dieuleveut 2010, Gunnell 2011)

Ceuta forma parte del eje del Estrecho de Gibraltar que ha tenido un papel muy importante en el flujo genético y/o barrera migratoria de algunas especies de quirópteros dando lugar a determinados patrones filogeográficos en especies como Myotis punicus/M. myotis/M. blythii o Eptesicus isabelinus/E. serotinus, separando genéticamente poblaciones del norte de África con las peninsulares (Arlettaz et al 1997, Castella et al 2000, Beuneux 2004, Garcia-Mudarra et al 2009, Juste et al 2009, Biollaz et al 2010). Algunos estudios genéticos han tratado de interpretar el papel de el Estrecho de Gibraltar como paso en la ruta migratoria y su papel durante la expansión de algunas especies después de la era glacial como Miniopterus schreibersii (Pereira et al 2009). Se desconoce también si actualmente sirve como paso migratorio o no para las colonias establecidas en Ceuta. De hecho, se cree que para algunas especies el Estrecho actúa a modo de barrera geográfica.

Recientemente, desde el Departamento de Medio Ambiente de Ceuta y la Empresa Prointec, por encargo del Gobierno de Ceuta, se está elaborando el Plan de Ordenación de los Recursos Naturales y Gestión (PORNG) del espacio LICCalamocarro-Benzú. En el marco de las convocatorias de ayudas a la Investigación del Instituto de Estudios Ceutíes, y debido a esta necesidad de actualizar el conocimiento de la situación de las colonias de cría refugiadas en Ceuta, así como para ampliar el inventario con las especies no cavernícolas, este proyecto se focalizó en dos objetivos:

1. Realizar una revisión exhaustiva de todos los refugios conocidos y potenciales en vista a poder planificar un seguimiento protocolizado de las colonias a largo plazo.

2. Realizar muestreos en las zonas forestales de la Ciudad Autonómica de Ceuta, en pantanos y en charcas para poder complementar el inventario con especies no-cavernícolas no detectadas con anterioridad (sesiones de captura + estaciones automáticas de grabación de ultrasonidos).

\section{MATERIALES Y MÉTODOS}

\section{AREA de ESTUdio: LIC-ZEPA CALAMOCARRo-BenZú}

De todo el territorio objeto de estudio destaca la zona de Calamocarro-Benzú, que ha sido propuesta como Lugar de Importancia Comunitaria (ES6310001) y Zona de Especial Protección para las Aves (ZEPA). Se emplaza junto a la misma Ciudad de Ceuta, ocupando una superficie de 601,81 ha en un espacio donde la altitud varía desde el nivel del mar a 349 $\mathrm{m}$ de altitud. Presenta un ombroclima de tipo subhúmedo, de alrededor de $600 \mathrm{~mm}$ de precipitación anual. Se debe mencionar la importancia de las criptoprecipitaciones en la zona, que ayudan a compensar el déficit hídrico estival, así como fenómenos como el rocía, nieblas y brumas en invierno. En verano, la etapa con déficit hídrico tiene lugar entre Junio y Octubre.

En relación a la geología de esta zona aparecen áreas con filitas, calizas y con dolomías. Se caracteriza por presentar pendientes elevadas (20-50\%)con numerosos arroyos y barrancos. La zona está sufriendo un proceso de cambio como consecuencia del abandono casi total de la actividad agropecuaria con una recuperación espontánea de la vegetación natural y con evidencias de repoblación primaria de la vegetación. Los cauces más representativos de esta zona vierten sus aguas a la costa norte en la Mediterránea: el Barranco de Benzú, el Barranco de Calamocarro, el Barranco del Renegado y el Barranco del Infierno. Se tiene que señalar que tanto el Barranco del Renegado como el Barranco del Infierno están regulados por sendas presas. Como hábitat especialmente importante, no incluido en el Anexo I de la Directiva 92/43/CEE, destaca la comunidad Rupicapnetum africanae, la única formación calcícola exclusivamente norteafricana de todo el territorio español y de la Unión Europea. Finalmente, mencionar que del $48 \%$ indicado como 
monocultivos forestales, una tercera parte está integrada por pinares (Pinus sp.). Además, en determinadas zonas ocupadas por matorral puede constatarse la presencia de alcornoques (Quercus suber) dispersos, alrededor de los cuales existe una regeneración natural del alcornocal.

Sesiones de capturas: Las sesiones de capturas se realizaron mediante redes de niebla "Ecotone", de 3 a 18 metros durante la última semana del mes de Junio de 2011. De los animales capturados se tomaron las medidas estándar, procediéndose a la identificación de las especies, su edad y su estado reproductor tan rápidamente como fuera posible. Todos los animales fueron liberados tras su manipulación en la misma localidad y durante la misma noche en la que fueron capturados. Se realizaron sesiones de capturas en las siguientes localidades: Arroyo Calamocarro (30S 286855, 3976897), Pinar del Castillo del Desnarigado (30S 293959, 3975407), Baterías de Valdeaguas (30S 293203, 3975791), Pinar del Fuerte de Aranguren (30S 285913, 3976032), Embalse del Infierno Charca (30S 288457, 3974846). Las redes se dispusieron de modo que se considerara más eficaz en cada localidad por su propia distribución. Todas las sesiones de captura fueron complementadas con las detecciones de murciélagos con detectores manuales de los modelos Petterson D240X.

Detección de quirópteros con detectores: Para ampliar el inventario y poder valorar los grados de actividad de las diferentes áreas muestreadas se instalaron algunas estaciones automáticas de grabación de ultrasonidos en localidades distintas cada noche. Se usaron detectores automáticos del modelo Petterson D500X, y los datos fueron analizados con el software Avisoft SAS Lab Pro y BatSound. La identificación de las grabaciones fue realizada manualmente dado el poco número de pistas acumuladas. La identificación se realizó básicamente con la frecuencia fundamental y la forma de los pulsos, mediante la comparación directa con librerías de referencia propias. Las especies que no pudieron ser clasificadas hasta nivel de especie se clasificaron en grupos fónicos como por ejemplo Myohigh o piplow. Los detectores estuvieron activos toda la noche, aproximadamente entre 570 y 600 horas en continuo con la siguiente cofiguración: Manual recording; Input gain: HET (left / right); Phones: normal; MAN; 384kHz; TE 10; Pre $2 \mathrm{~s}$

Post 3 s; Play man; Auto save; Trigger HF; HP OFF. Se instalaron detectores automáticos en las siguientes localidades las noches de muestreo: Torre de la Huerta de Regulares (30S 286295, 3976337), Charca del Embalse del Infierno (30S 288457, 3974846), Embalse del Renegado, Acantilados del Monte Hacho (30S 293919, 3974641).

Revisión de refugios: Las revisiones de los refugios fueron realizadas mediante revisión y recuento visual, valoración del número de individuos en cada refugio, y valoración de la cantidad de heces y rastros localizados. Para ver la lista de los refugios revisados ver Tabla 2. Se consideraron y revisaron todos aquellos refugios de los que se tenía constancia de la presencia de murciélagos en años anteriores, más un conjunto de refugios potenciales que fueron compilados previamente mediante una revisión exhaustiva de instalaciones y localidades repartidas en la Comunidad Autonómica de Ceuta.

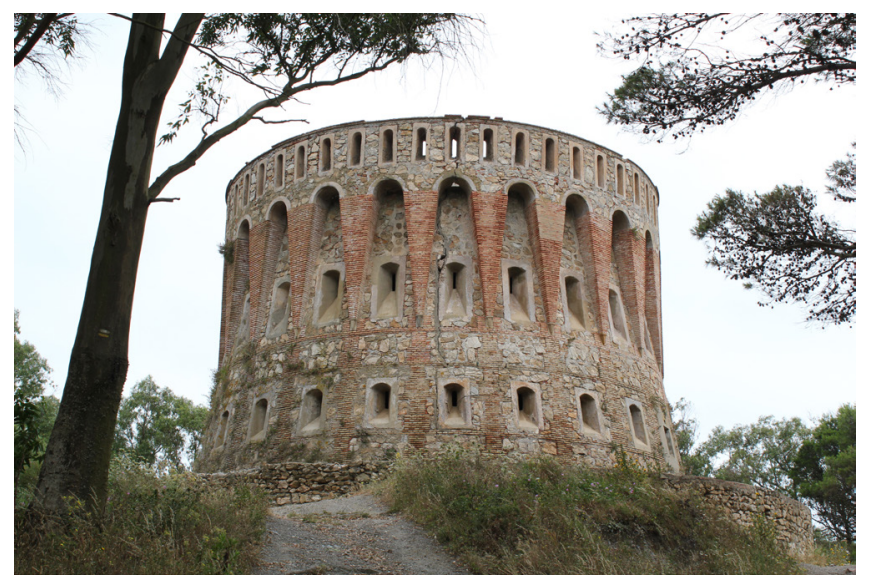

Fig. 1. Estructura típica de los fuertes presentes en los parajes de Ceuta (Fuerte de Isabel II)

\section{Resultados}

Se localizaron refugios de cría de cría solamente de la especie Rhinolophus hipposideros en los refugios siguientes: Fuerte de Pinier, Fuerte de Anyera y Torre de la Fuente de la Higuera.

En el Fuerte de Pinier la colonia fue localizada en el espacio central de la torre. Se trata de una fortificación completamente tapiada, pero con algunos orificios por los que se puede acceder. En estos momentos, este Fuerte se encuentra desocupado.

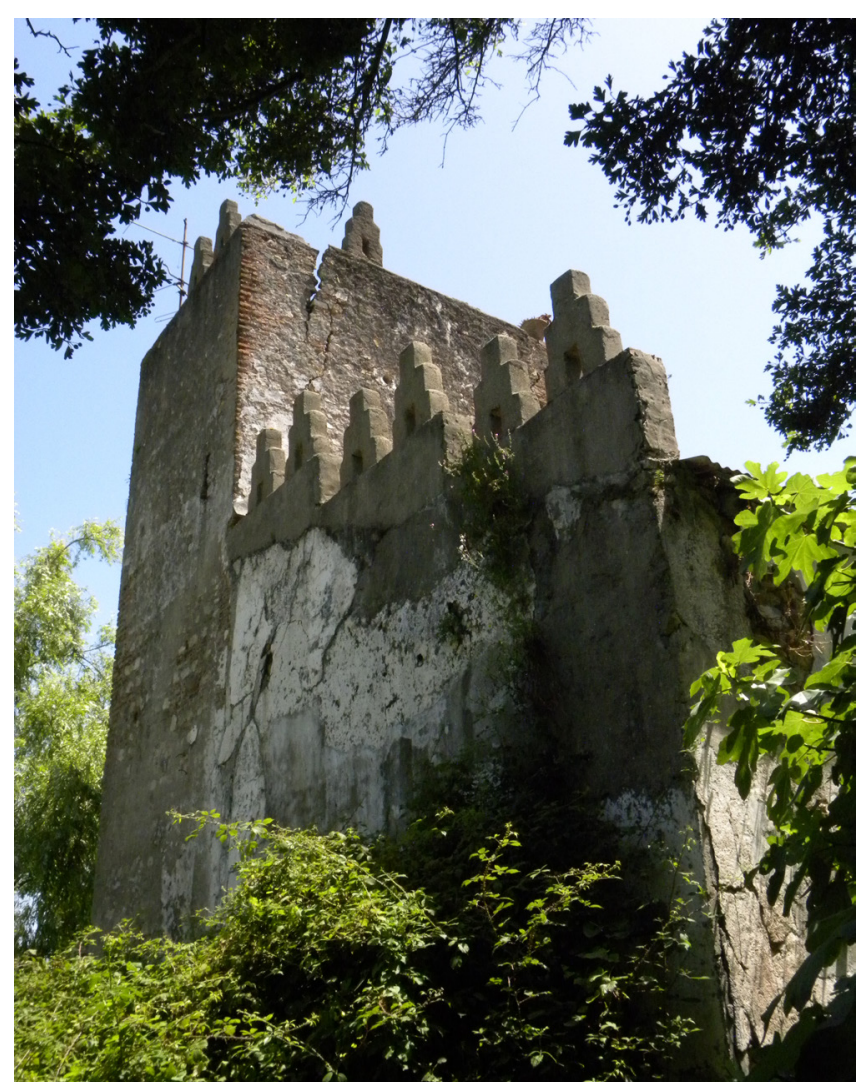

Fig. 2. Torre de la Fuente de la Higuera

En el Fuerte de Anyera la colonia se refugiaba en el piso inferior del edificio. Como la anterior, se trata de una fortificación abandonada y en mal estado de conservación, con un pozo con agua en su interior. No se detectaron individuos en el interior del pozo. En las paredes laterales de la misma fortificación existen unas pequeñas cámaras de 
Detección de quirópteros mediante detectores

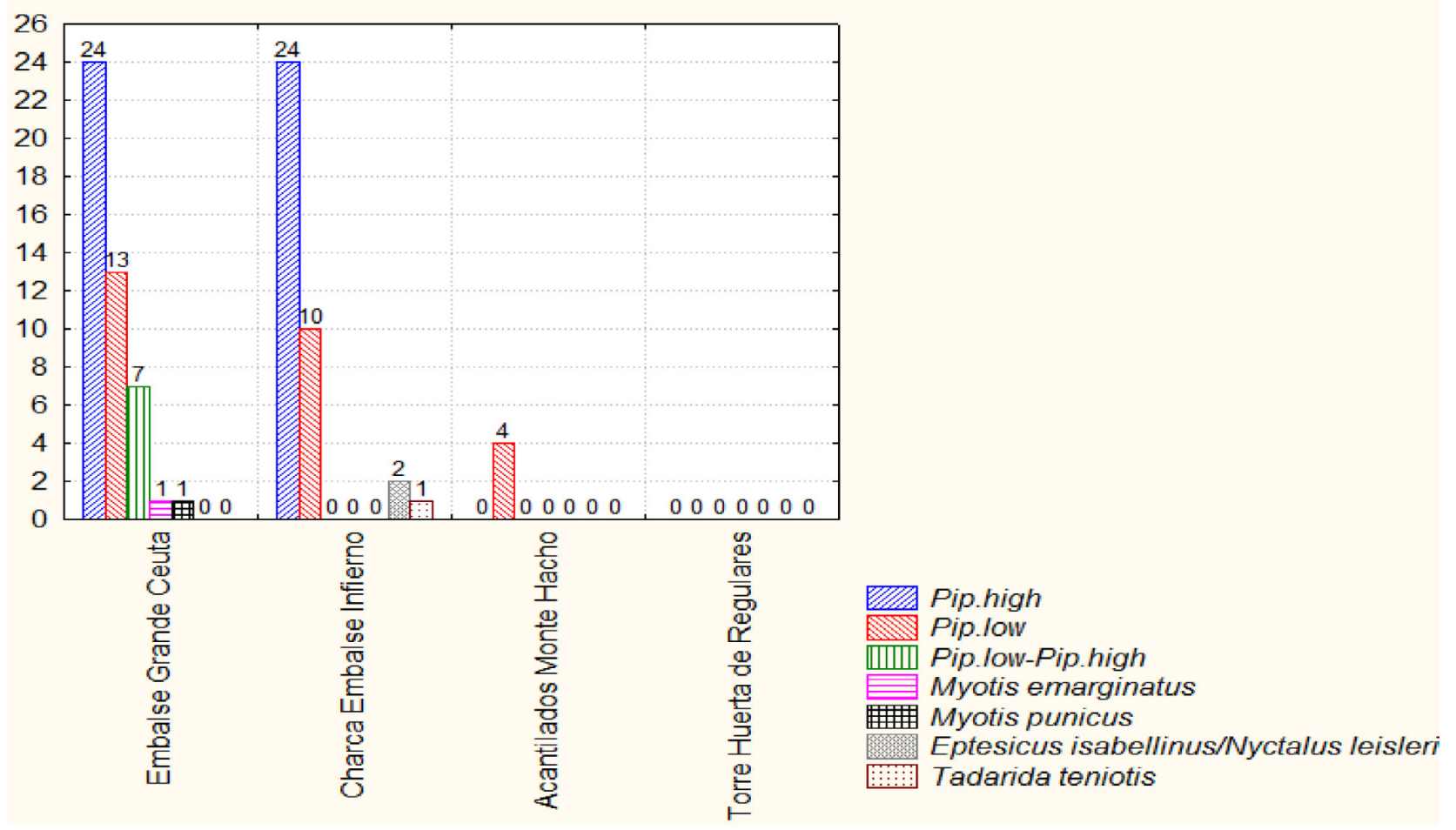

Fig. 3. Número total de detecciones mediante grabación automática.

aire donde se refugiaban algunas hembras de $R$. hipposideros junto con sus crías. Se trata de un refugio compartido con ejemplares aislados de la especie $R$. ferrumequinum.

La Torre de la Fuente de la Higuera es un edificio de 3 pisos completamente abandonado y semiderrumbado donde fue localizada otra colonia de $R$. hipposideros. Como en el Fuerte de Anyera la colonia compartía refugio con al menos un ejemplar de $R$. ferrumequinum. Todos los ejemplares, fueron localizados en el $3 \mathrm{r}$ piso, en un tejado de madera, mientras que los excrementos se encontraban esparcidos por todo el habitáculo en abundancia.

Individuos aislados de Rhinolophus ferrumequinum (4 ejemplares) fueron capturados y localizados en el Fuerte del Monte del Renegado o Túneles de la Tortuga, Fuerte de Anyera y Torre de la Fuente de la Higuera. En Cambio, Myotis punicus (1 ejemplar), solamente fue capturado en la Mina de la Fuente, siendo el único ejemplar remanente en este refugio.

De todas las zonas muestreadas mediante redes de niebla se capturaron quirópteros únicamente en la Charca del Embalse del Infierno, siendo Pipistrellus pygmaeus la única especie capturada con 3 individuos $(2$ hembras lactantes y un macho). P. pygmaeus ha sido identificado morfológicamente siguiendo Dietz et al (2009). En la misma estación de capturas se detectó un ejemplar de Tadarida teniotis mediante el detector Petterson D240X (grabadora digital Edirol). Tadarida teniotis fue igualmente detectada en el Arroyo de Calamocarro emitiendo sonidos característicos entre $10-14 \mathrm{Khz}$.

\section{Discusión}

Entre las últimas revisiones de refugios efectuadas en Ceuta el 2001 y 2004 y las actuales ha habido una pérdida de especies y de refugios. Se tenía constancia de 7 especies de murciélagos incluidas en el Anexo II de la Directiva Hábitats 92/43/CEE: Miniopterus schreibersii, Myotis capaccinii, Rhinolophus blasii, Rhinolophus euryale, Rhinolophus ferrumequinum, Rhinolophus hipposideros y Rhinolophus mehelyi, de las que en 2011 solamente se han detectado 3. Sin duda, el derrumbamiento natural de la entrada de la Mina de la Fuente ha provocado un movimiento de los murciélagos que se refugiaban allí a otros refugios. Afortunadamente el desprendimiento dejó un espacio abierto en la entrada de la cueva por donde pudieron escapar los ejemplares que posiblemente quedaron atrapados. Se trataba de una cueva con presencia de 4 especies distintas, dos de las cuales clasificadas como amenazadas (Rhinolophus mehelyi y Miniopterus schreibersii). Por otra parte, las citas de Myotis capaccinii y Rhinolophus blasii en otros refugios no pudieron ser confirmadas. Dada la actual distribución de Rhinolophus blasii, parece improbable su presencia en la ciudad autónoma de Ceuta. De hecho, la única cita en la lista de referencia española se localiza en Ceuta, es del 1997 y su autor la considera dudosa.

Una de las causas del declive de las poblaciones de los quirópteros cavernícolas probablemente fue la elevada mortandad en 1993 de individuos que fueron sacrificados para la realización de análisis rábicos (Serra Cobo 1993). Más concretamente, en la Mina de la Fuente hay conocimiento que en 1993 se encontró una colonia de 10 Rhinolophus mehelyi y otra de 30 ejemplares de Myotis punicus que fueron sacrificados; Individuos de estas especies no han sido localizados de nuevo a posteriori en ninguna de las 


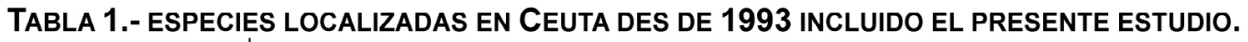

\begin{tabular}{|c|c|c|c|c|c|c|c|c|}
\hline Especie & Localidad & Estación & Año & Observador & Macho & Hembra & Ind. & Obs. \\
\hline Rhinolophus euryale & Mina de la Fuente & & 1993 & J. Serra-Cobo & 1 & 0 & 0 & \\
\hline \multirow{2}{*}{ Rhinolophus mehelyi } & Mina de la Fuente & & 1993 & J. Serra-Cobo & 10 & 0 & 0 & \\
\hline & Mina de la Fuente & & 1997 & Carlos Ibáñez et al & 0 & 0 & 3 & \\
\hline \multirow{7}{*}{$\begin{array}{l}\text { Rhinolophus } \\
\text { hipposideros }\end{array}$} & Túneles Tortuga & & & $\begin{array}{l}\text { Comunicación } \\
\text { verbal }\end{array}$ & 0 & 0 & 1 & \\
\hline & Torre la Higuera & Junio & 1996 & $\begin{array}{l}\text { Francisco Ja } \\
\text { Martínez }\end{array}$ & 0 & 0 & 1 & \\
\hline & Mina de la Fuente & Mayo & 2001 & Carlos Ibáñez et al & 0 & 0 & 2 & \\
\hline & Fuerte Isabel II & Mayo & 2001 & Carlos Ibáñez et al & 0 & 16 & 0 & Cría \\
\hline & Torre la Higuera & Junio & 2011 & Museu Granollers & 0 & 5 & 0 & Cría \\
\hline & Fuerte de Anyera & Junio & 2011 & Museu Granollers & 0 & 7 & 0 & Cría \\
\hline & Fuerte Pinier & Junio & 2011 & Museu Granollers & 0 & 12 & 0 & Cria \\
\hline \multirow{6}{*}{$\begin{array}{l}\text { Rhinolophus } \\
\text { ferrumequinum }\end{array}$} & Mers d'Aulud & & 1993 & J. Serra-Cobo & 7 & 0 & 0 & \\
\hline & Búnquer la Emisora & & 1993 & J. Serra-Cobo & 9 & 0 & 0 & \\
\hline & Fuerte de Anyera & Julio & 2001 & $\begin{array}{l}\text { Francisco } \mathrm{J}^{\mathrm{a}} \\
\text { Martínez }\end{array}$ & 0 & 0 & 1 & (4 sin id.) \\
\hline & Túneles Tortuga & Junio & 2011 & Museu Granollers & 1 & 0 & 0 & \\
\hline & Torre la Higuera & Junio & 2011 & Museu Granollers & 0 & 0 & 1 & \\
\hline & Fuerte de Anyera & Junio & 2011 & Museu Granollers & 0 & 0 & 2 & \\
\hline Rhinolophus blasii & Castillo de Aranguren & Mayo & 1997 & $\begin{array}{l}\text { Francisco } \mathrm{J}^{\mathrm{a}} \\
\text { Martínez }\end{array}$ & 0 & 0 & 1 & Cita dudosa \\
\hline \multirow{2}{*}{ Miniopterus schreibersii } & Mina de la Fuente & & 1997 & $\begin{array}{l}\text { Francisco Ja } \\
\text { Martinez }\end{array}$ & 0 & 0 & 1 & \\
\hline & Mina de la Fuente & Octubre & 2004 & $\begin{array}{l}\text { Francisco Ja } \\
\text { Martínez }\end{array}$ & 0 & 0 & $?$ & \\
\hline \multirow{8}{*}{ Myotis punicus } & Mina de la Fuente & & 1993 & J. Serra-Cobo & 11 & 19 & 0 & \\
\hline & Mers d'Aulud & & 1993 & J. Serra-Cobo & 1 & 1 & 0 & \\
\hline & Mina Afriquita & & 1993 & J. Serra-Cobo & 1 & 0 & 0 & \\
\hline & Mina de la Fuente & Mayo & 1997 & Carlos Ibáñez et al & 0 & 0 & 19 & \\
\hline & Mina de la Fuente & & 2000 & Castella et al & 13 & 6 & 0 & \\
\hline & Mina de la Fuente & & 2001 & Carlos Ibáñez et al & 0 & 0 & 8 & \\
\hline & Mina de la Fuente & Octubre & 2004 & $\begin{array}{l}\text { Francisco Ja } \\
\text { Martínez }\end{array}$ & 0 & 0 & $?$ & \\
\hline & Mina de la Fuente & Junio & 2011 & Museu Granollers & 1 & 0 & 0 & \\
\hline Myotis capaccinii & Castillo de Aranguren & Mayo & 1997 & $\begin{array}{l}\text { Francisco } \mathrm{J}^{\mathrm{a}} \\
\text { Martínez }\end{array}$ & 0 & 0 & 1 & \\
\hline Myotis emarginatus (?) & Embalse del Renegado & Junio & 2011 & Museu Granollers & 0 & 0 & 1 & $\begin{array}{l}\text { Cita con } \\
\text { detector }\end{array}$ \\
\hline $\begin{array}{l}\text { Eptesicus isabellinus } \\
\text { Nyctalus leisleri }\end{array}$ & $\begin{array}{l}\text { Charca embalse del } \\
\text { Infierno }\end{array}$ & Junio & 2011 & Museu Granollers & 0 & 0 & 1 & $\begin{array}{l}\text { Cita con } \\
\text { detector }\end{array}$ \\
\hline Pipistrellus pipistrellus & Ceuta ciudad & & 1994 & $\begin{array}{l}\text { Francisco Ja } \\
\text { Martínez }\end{array}$ & 0 & 0 & 1 & \\
\hline Pipistrellus pygmaeus & $\begin{array}{l}\text { Charca Embalse } \\
\text { Infierno }\end{array}$ & Junio & 2011 & Museu Granollers & 1 & 2 & 0 & \\
\hline Tadarida teniotis & $\begin{array}{l}\text { Charca Embalse } \\
\text { Infierno }\end{array}$ & Junio & 2011 & Museu Granollers & 0 & 0 & 1 & \\
\hline
\end{tabular}


TABLA 2.- DATOS DE REVISIONES DE REFUgIOS DES DE 1993 INCLUIDO EL PRESENTE ESTUDIO

\begin{tabular}{|c|c|c|c|c|c|c|c|c|}
\hline Localidad & Estación & Año & Observador & Macho & Hembra & Ind. & Especie & Obs. \\
\hline Baterías de Valdeaguas & Junio & 2011 & Museu Granollers & 0 & 0 & 0 & & \\
\hline Búnquer la Emisora & & 1993 & J. Serra-Cobo & 9 & 0 & 0 & Rhinolophus ferrumequinum & \\
\hline Castillo del Desnarigado & Junio & 2011 & Museu Granollers & 0 & 0 & 0 & & \\
\hline $\begin{array}{l}\text { Cueva de la Cantera de } \\
\text { Benzú }\end{array}$ & Junio & 2011 & Museu Granollers & 0 & 0 & 0 & & \\
\hline Cueva y Abrigo de Benzú & Junio & 2011 & Museu Granollers & 0 & 0 & 0 & & \\
\hline $\begin{array}{l}\text { ETAP ACEMSA } \\
\text { (Bda Postigo) }\end{array}$ & Junio & 2011 & Museu Granollers & 0 & 0 & 0 & & \\
\hline \multirow{3}{*}{ Fuerte de Anyera } & Julio & 2001 & $\begin{array}{l}\text { Francisco } \mathrm{J}^{\mathrm{a}} \\
\text { Martínez }\end{array}$ & 0 & 0 & 1 & Rhinolophus ferrumequinum & (4 sin id.) \\
\hline & Junio & 2011 & Museu Granollers & 0 & 7 & 0 & Rhinolophus hipposideros & Cría \\
\hline & Junio & 2011 & Museu Granollers & 0 & 0 & 2 & Rhinolophus ferrumequinum & \\
\hline \multirow{3}{*}{ Fuerte de Aranguren } & Mayo & 1997 & $\begin{array}{l}\text { Francisco Ja } \\
\text { Martínez }\end{array}$ & 0 & 0 & 1 & Rhinolophus blasii & $\begin{array}{l}\text { Cita } \\
\text { dudosa }\end{array}$ \\
\hline & Mayo & 1997 & $\begin{array}{l}\text { Francisco Ja } \\
\text { Martínez }\end{array}$ & 0 & 0 & 1 & Myotis capaccinii & \\
\hline & Junio & 2011 & Museu Granollers & 0 & 0 & 0 & & \\
\hline Fuerte de Mendizábal & Junio & 2011 & Museu Granollers & 0 & 0 & 0 & & \\
\hline Castillo de Monte Hacho & Junio & 2011 & Museu Granollers & 0 & 0 & 0 & & \\
\hline Fuerte Francisco de Asís & Junio & 2011 & Museu Granollers & 0 & 0 & 0 & & \\
\hline Fuerte Isabel II & Mayo & 2001 & Carlos lbáñez et al & 0 & 16 & 0 & Rhinolophus hipposideros & Cría \\
\hline Fuerte Pinier & Junio & 2011 & Museu Granollers & 0 & 12 & 0 & Rhinolophus hipposideros & Cría \\
\hline La Sirena & Junio & 2011 & Museu Granollers & 0 & 0 & 0 & & \\
\hline \multirow{2}{*}{ Mers d'Aulud } & & 1993 & J. Serra-Cobo & 7 & 0 & 0 & Rhinolophus ferrumequinum & \\
\hline & & 1993 & J. Serra-Cobo & 1 & 1 & 0 & Myotis punicus & \\
\hline Mina Afriquita & & 1993 & J. Serra-Cobo & 1 & 0 & 0 & Myotis punicus & Tapiada \\
\hline \multirow{13}{*}{ Mina de la Fuente } & & 1993 & J. Serra-Cobo & 1 & 0 & 0 & Rhinolophus euryale & \\
\hline & & 1993 & J. Serra-Cobo & 10 & 0 & 0 & Rhinolophus mehelyi & \\
\hline & & 1993 & J. Serra-Cobo & 11 & 19 & 0 & Myotis punicus & \\
\hline & Mayo & 1997 & Carlos Ibáñez et al & 0 & 0 & 19 & Myotis punicus & \\
\hline & Mayo & 1997 & Carlos lbáñez et al & 0 & 0 & 3 & Rhinolophus mehelyi & \\
\hline & & 1997 & $\begin{array}{l}\text { Francisco Ja } \\
\text { Martínez }\end{array}$ & 0 & 0 & 1 & Miniopterus schreibersii & \\
\hline & & 2000 & Castella et al & 13 & 6 & 0 & Myotis punicus & \\
\hline & Mayo & 2001 & Carlos lbáñez et al & 0 & 0 & 2 & Rhinolophus hipposideros & \\
\hline & Mayo & 2001 & Carlos Ibáñez et al & 0 & 0 & 8 & Myotis punicus & \\
\hline & Octubre & 2004 & $\begin{array}{l}\text { Francisco Ja } \\
\text { Martínez }\end{array}$ & 0 & 0 & $?$ & Miniopterus schreibersii & \\
\hline & Octubre & 2004 & $\begin{array}{l}\text { Francisco } \mathrm{J}^{\mathrm{a}} \\
\text { Martínez }\end{array}$ & 0 & 0 & $?$ & Myotis punicus & \\
\hline & Junio & 2006 & Nadia Bruyndonckx & 0 & 0 & 0 & & \\
\hline & Junio & 2011 & Museu Granollers & 1 & 0 & 0 & Myotis punicus & \\
\hline \multirow{3}{*}{ Torre la Higuera } & Junio & 1996 & $\begin{array}{l}\text { Francisco } \mathrm{J}^{\mathrm{a}} \\
\text { Martínez }\end{array}$ & 0 & 0 & 1 & Rhinolophus hipposideros & \\
\hline & Junio & 2011 & Museu Granollers & 0 & 5 & 0 & Rhinolophus hipposideros & Cría \\
\hline & Junio & 2011 & Museu Granollers & 0 & 0 & 1 & Rhinolophus ferrumequinum & \\
\hline Torre Regulares & Junio & 2011 & Museu Granollers & 0 & 0 & 0 & & \\
\hline Túneles de Santa Catalina & Junio & 2011 & Museu Granollers & 0 & 0 & 0 & & \\
\hline \multirow[t]{2}{*}{ Túneles Tortuga } & & & $\begin{array}{l}\text { Comunicación } \\
\text { verbal }\end{array}$ & 0 & 0 & 1 & Rhinolophus hipposideros & \\
\hline & Junio & 2011 & Museu Granollers & 1 & 0 & 0 & Rhinolophus ferrumequinum & \\
\hline
\end{tabular}


prospecciones en esta mina por parte de distintos equipos de investigación. Paralelamente, otra de las causas de la dificultad de recuperación frente este declive de especies podría ser la continua degradación de los hábitats de la zona entre 1940-1960, que se convirtieron en bosques primarios, básicamente compuestos por pinares monoespecíficos con muy poco sotobosque y en proceso de regeneración. A fecha de hoy, se observa una mejora en la calidad de los hábitats, pero que posiblemente aun no se refleje en la actividad y diversidad de quirópteros.

Las únicas colonias de cría que se han localizado corresponden a Rhinolophus hipposideros, una especie cavernícola bastante oportunista y adaptada a ambientes antropizados. De todos modos, la cantidad de hembras con cría detectada en estas sesiones de 2011 se considera relativamente abundante y merecedora de especial atención. Uno de los refugios (Torre de la Higuera), que está situado en el área del LIC - Calamocarro Benzú, fue considerado en una propuesta de conservación de fauna como refugio protegido para especies cavernícolas. La propuesta planteó conflictos entre su interés faunístico y su conservación como Bien de Interés Cultural.

Las únicos individuos capturados en abrevaderos con redes de niebla pertenecen a la especie Pipistrellus pygmaeus, en lo que corresponde a la primera cita de la especie en el continente africano y confirma la presencia de colonias de cría en el territorio. Se amplia, pues, el conocimiento sobre la distribución de la especie, que fue capturada en humedales cercanos a un embalse.

En relación a los muestreos de especies forestales, ha quedado plasmada la pobreza de especies en este tipo de ambientes en el área de Ceuta. Las únicas especies detectadas mediante detección automática y sesiones de captura corresponden a especies de carácter antrópico y claramente oportunista como Pipistrellus pygmaeus, P. kulhii/P.pipistrellus, Eptesicus isabellinus/Nyctalus leisleri, Tadarida teniotis. No se han detectado especies estrictamente forestales, y los niveles de actividad en los bosques han sido extremadamente bajos o nulos en toda la noche en la Torre de Regulares hasta un máximo de 45 detecciones; incluso en puntos de atracción como abrevaderos.

Del mismo modo en la zona de los Acantilados del Monte Hacho, la actividad de quirópteros detectada fue muy pobre, y correspondió perfectamente con la sesión de capturas en el pinar de la zona, donde no se capturó ningún ejemplar ni se detectó ningún quiróptero mediante detectores manuales. Probablemente las especies presentes, de naturaleza antrópica se refugien directamente en el casco urbano de Ceuta, y se desplacen asimismo al LIC Calamocarro-Benzú, que hay mayor disponibilidad de agua y fragmentos de bosque de mayor dimensión. En general la aridez y extrema humanización del territorio parece provocar la pobreza de fauna quiropterològica observada.

De todos modos, la información que se tiene de toda esta área desde sus primeros muestreos en 1993 es claramente deficitaria, puesto que los lapsos temporales entre prospecciones son demasiado extensos, sin ningún tipo de regularidad ni protocolo; por lo que tratar de inferir causas de la disminución de quirópteros cavernícolas es una tarea de poca consistencia en cuanto a la gestión actual para la conservación de estas comunidades. En consecuencia, y dada la facilidad de acceso a los refugios y la rapidez con la que se podría realizar, se propone la aplicación de un seguimiento monitorizado anual de refugios. A la luz de los resultados se considera prioritario el seguimiento continuado de las colonias reproductoras de Rhinolophus hipposideros, dado su mayor número de individuos en el área, juntamente con un incremento de protección de las estructuras que usan como refugio (considerando asimismo fuertes, fortificaciones y torres abandonadas) evitando el acceso desde el exterior.

Del mismo modo, sería recomendable la realización de sesiones de muestreo en época primaveral y otoñal para determinar que especies usan estos espacios naturales como ambientes de paso, usando sus recursos hídricos y como zona de caza durante sus rutas migratorias. En general se echan en falta citas de especies que por su distribución y hábitos deberían ser presentes en Ceuta, como el murciélago de borde claro (Pipistrellus kuhlii) y el murciélago montañero (Hypsugo savii). Además, sería muy importante una evaluación de la posible recolonización de la Mina de la Fuente, que ha sido abierta de nuevo durante este Junio de 2011, para determinar si las colonias antiguamente detectadas de Rhinolophus euryale, Rhinolophus mehelyi, Miniopterus schreibersii y Myotis punicus regresan o no a la mina.

\section{Agradecimientos}

Quisiéramos agradecer la ayuda que nos brindó Francisco Javier Martínez Medina (de la empresa Prointec), a Carlos Ibáñez (del CSIC de Doñana) por la información previa y Toni Arrizabalaga (del Museo de Granollers) por su ayuda en la gestión del proyecto.

Agradecer igualmente a la Comandancia General de Ceuta, la Confederación Hidrográfica del Guadalquivir y ACEMSA por las facilidades prestadas para acceder a sus instalaciones.

Este proyecto fue realizado con una beca de la Convocatoria de Ayudas a la Investigación del año 2010, promovida por el Instituto de Estudios Ceutíes.

\section{REFERENCIAS}

ArlettaZ, R., Ruedi. M. 1997. “A new perspective on the zoogeography of the sibling mouse-eared bat species Myotis myotis and Myotis blythii: Morphological, genetical and ecological evidence." Journal of Zoology 242: 45-62. DOI: https://dx.doi. org/10.1111/j.1469-7998.1997.tb02928.X

Beuneux, G. 2004. "Morphometrics and ecology of Myotis cf. punicus (Chiroptera, Vespertilionidae) in Corsica." Mammalia 68(4): 269-273. DOI: https://dx.doi. org/10.1515/mamm.2004.025

Biollaz, F., BruYndoncKx, N. 2010. "Genetic isolation of insular populations of the Maghrebian bat, Myotis punicus, in the Mediterranean Basin." Journal of Biogeography 37(8): 1557-1569.

Castella, V., Ruedi, M. 2000. “Is the Gibraltar Strait a barrier to gene flow for the bat Myotis myotis (Chiroptera : Vespertilionidae)?" Molecular Ecology 9(11): 1761-1772. 
Dietz, C., von Helversen, O. \& Nill, D. 2009. Bats of Britain, Europe and Northwest Africa. London: A. C. Black

Dieuleveut, T., Lieron, V., Hingrat, Y. 2010 Nouvelles données sur la repartition des Chiroptères dans le Maroc oriental (années 2007 à 2009). Bulletin de l'Institut Scientifique de la Vie 32(1): 33-40

Garcia-Mudarra, J. L., Ibanez. C. 2009. "The Straits of Gibraltar: barrier or bridge to Ibero-Moroccan bat diversity?" Biological Journal of the Linnean Society 96(2): 434-450. DOI: https://dx.doi. org/10.1111/j.1095-8312.2008.01128.x

Gunnell, G. F., Eiting, T. P. 2011. "New late Pliocene bats (Chiroptera) from Ahl al Oughlam, Morocco." Neues Jahrbuch Fur Geologie Und PalaontologieAbhandlungen 260(1): 55-71. DOI: https://dx.doi. org/10.1127/0077-7749/2011/0128

Juste, J., BILGIN, R. 2009. "Mitochondrial DNA signatures at different spatial scales: from the effects of the Straits of Gibraltar to population structure in the meridional serotine bat (Eptesicus isabellinus)." Heredity 103(2): 178-187. DOI: https://dx.doi. org/10.1038/hdy.2009.47
Lieron, V., Poulouin, E., Amezian, M., Qninba, A., Thevenot, M. 2008 Inventaire des Chiroptères de l'arrière-pays du port Tanger-Méditerranée (NordOuest du Maroc). Bulletin de l'Institut Scientifique, rabat, section Science de la Vie 30(1): 53-54

Pereira, M. J. R., Salgueiro, P. 2009. "Population Structure of a Cave-Dwelling Bat, Miniopterus schreibersii: Does It Reflect History and Social Organization?" Journal of Heredity 100(5): 533-544. DOI: https:// dx.doi.org/10.1093/jhered/esp032

Plan de Ordenación de Recursos Naturales y de Gestión del LIC-ZEPA Calamocarro - Benzú (Ceuta)

SERrA-Cobo, J. 1993. Impacto del virus rábico en los quirópteros españoles e interacción con la población humana. Universidad de Barcelona, Departamento de Biología animal

VIADA, C. 1998 Áreas Importantes para las Aves en España. Monografía $n^{\circ} 5$ de SEO/BirdLife 